\title{
Long term efficacy of drug coated balloon for radial artery angioplasty
}

\author{
Ahmed Mahmoud El Amrawy ${ }^{1,2}$, Javier León-Jiménez ${ }^{2}$, Santiago Jesus Camacho-Freire ${ }^{2}$, \\ Rosa Cardenal-Piris ${ }^{2}$, Jose F. Díaz-Fernández ${ }^{2}$ \\ ${ }^{1}$ Cardiology Department, Faculty of Medicine, Alexandria University, Egypt \\ ${ }^{2}$ Interventional Cardiology Department, Juan Ramon Jimenez University Hospital, Huelva, Spain
}

\begin{abstract}
A 76-year-old male patient, diabetic, hyperlipidemic, hypertensive, and ex-smoker, presented one year ago complaining of angina with $1 \mathrm{~mm}$ ST segment depressions in anterior chest leads. Coronary angiography was done via right radial approach. The patient had diffuse calcific stenosis in the right radial artery (Fig. 1A, B). As regards to coronaries, there was a proximal focal calcific lesion in the left anterior descending (LAD). A decision was taken for percutaneously treating LAD. The guiding catheter (6 Fr EBU3.5, Medrtonic, Danvers, USA) failed to advance through the stenosed radial artery, also a sheathless guiding catheter failed to advance. To overcome this obstacle, right radial angioplasty was done (Fig. 1C, D). A hydrophilic wire (Radifocus, Terumo, Tokyo, Japan) was used to cross and multiple inflations using peripheral paclitaxel drug coated balloon $4 \times 60 \mathrm{~mm}$
\end{abstract}

(Lutonix 035, BARD, Arizona, USA) were done at $4 \mathrm{~atm}$ with good angiographic result (Fig. 1E). The 6.5 Fr PB3.5 sheathless guiding catheter (Asahi Intecc, Aichi, Japan) was advanced easily through the radial artery till the ascending aorta to successfully engage the left main. Percutaneous intervention for calcific proximal LAD was then successfully performed.

One year later, the patient presented with recurrence of angina. During clinical examination, the right radial pulse was well felt. Coronary angiography was performed which revealed patent proximal LAD stent. The right radial artery was widely patent with minimal recoil (Fig. $1 \mathrm{~F}$ ).

It may be concluded that percutaneous radial angioplasty using drug coated balloons was a safe and effective tool, with an acceptable immediate technical success and good long term prognosis.

Conflict of interest: None declared

Address for correspondence: Ahmed Mahmoud El Amrawy, MD, Faculty of Medicine, Cardiology Department, Champlion Street, El Azarita, Alexandria, Egypt, Postal code: 21131, tel: +201006222922, fax: +2034862506, e-mail: dr.ahmed.elamrawy@hotmail.com

Received: $11.06 .2018 \quad$ Accepted: 16.06 .2018 


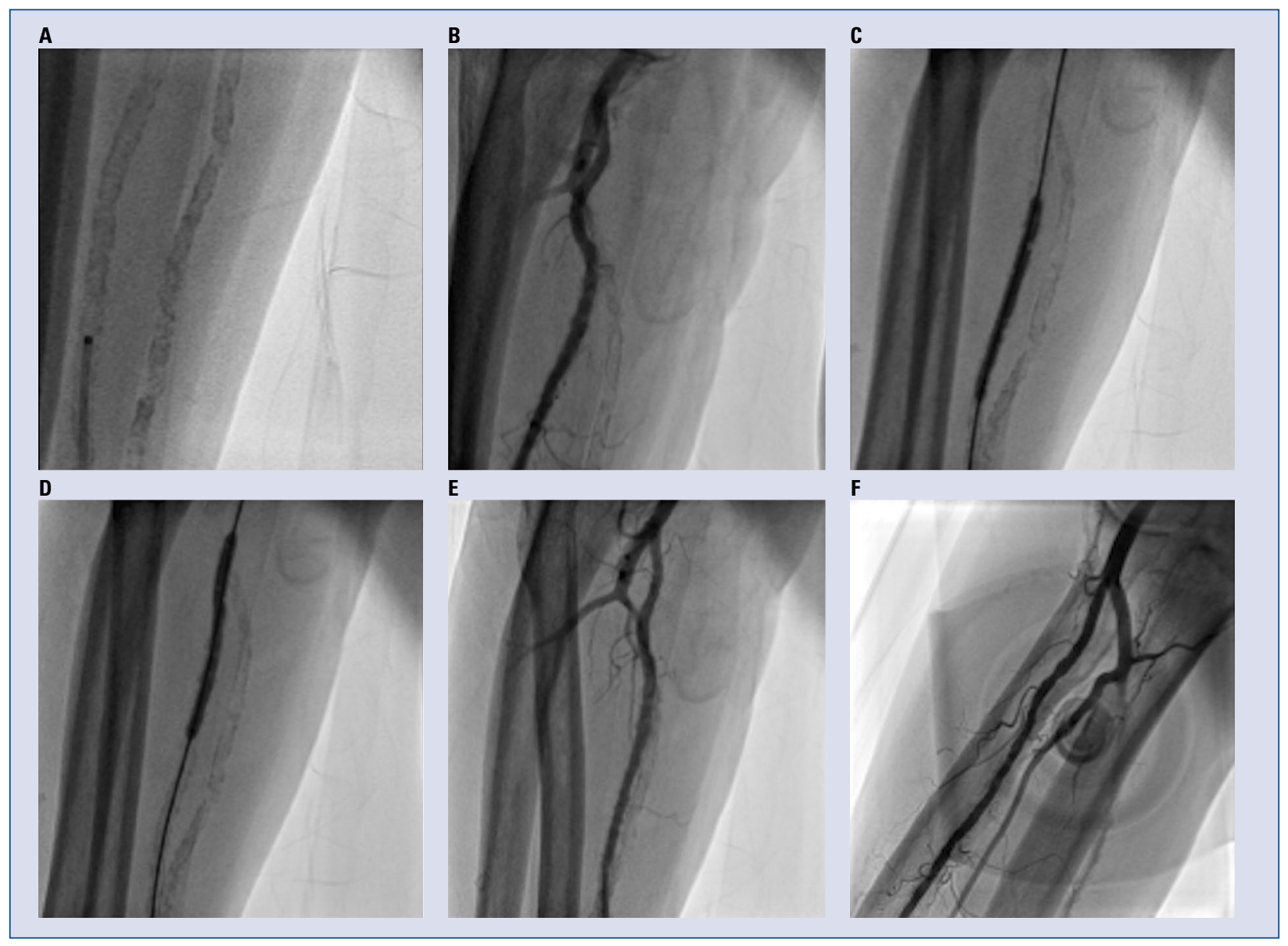

Figure 1. Angiogram showing: A, B. Heavily calcific right radial artery with multiple stenotic lesions; C, D. Radial angioplasty using peripheral paclitaxel drug coated balloon $4 \times 60 \mathrm{~mm}$ (Lutonix 035); E. Final angiographic result; F. Angiographic follow up after 1 year showed widely patent right radial artery with minimal recoil. 